\section{Does Pulmonary Involvement Play a Role in Right Ventricular Dysfunction of Patients with Idiopathic Inflammatory Myopathies?}

\section{To the Editor:}

We read with interest the article by Guerra, et al ${ }^{1}$ about subclinical cardiac systolic impairment in patients with idiopathic inflammatory myopathies (IIM). The researchers demonstrated that both left and right ventricular longitudinal strain of patients with IIM were significantly lower than their matched controls. Because they focused on IIM patients without overt clinical manifestation of heart disease, patients with pulmonary hypertension were excluded. In the subgroup analysis, pulmonary involvement was not associated with a reduced strain; however, although not significant, there were differences regarding the right ventricular global longitudinal strain (RVGLS): they were $-17.1 \%$ and $-20.9 \%$ in patients with and without lung involvement, respectively. We assume that this difference might have some implications. Even though the subclass of lung involvement was not specified in this study, the most common form of lung involvement in IIM was interstitial lung disease (ILD) ${ }^{2}$. In a study using cardiovascular (CV) magnetic resonance-derived right ventricular ejection fraction (RVEF) to define RV systolic function in patients with ILD, Kato, et $a l^{3}$ found RVEF was significantly lower in ILD patients without pulmonary hypertension than in healthy controls, while the underlying mechanism might be that systemic inflammation or pulmonary endothelial dysfunction influence the RV function in the early stage of ILD.

In addition to patients with ILD, subclinical cardiac dysfunction has been detected in populations with diabetes, dyslipidemia, and hypertension ${ }^{4,5,6}$, and these traditional $\mathrm{CV}$ risk factors are more prevalent in patients with IIM than in the general population ${ }^{7}$, so we cannot deny that reduced myocardial strain in patients with IIM is the interaction between comorbidities and inflammation/fibrosis in the myocardium, due to the disease per se. But speckle echocardiography still remains as the most effective screening method for cardiac dysfunction. The findings of reduced RVGLS may be seen as an asymptomatic state on the road to right heart failure. More followup studies are needed to establish the relationship between subclinical right heart dysfunction and the progress of ILD in patients with polymyositis and dermatomyositis
YUE ZHONG, MD; WENJUAN BAI, MD; LI RAO, MD, Department of Cardiology, West China Hospital of Sichuan University, Chengdu, China. Supported by the Science and Technology Pillar Program of Sichuan Province, Grant 2014SZ0004-8. Address correspondence to Dr. Rao, West China Hospital of Sichuan University, 37 Guo Xue Xiang, Chengdu, Sichuan 610041, China.E-mail: 1rlz1989@163.com

\section{REFERENCES}

1. Guerra F, Gelardi C, Capucci A, Gabrielli A, Danieli MG. Subclinical cardiac dysfunction in polymyositis and dermatomyositis: a speckle-tracking case-control study. J Rheumatol 2017;44:815-21.

2. Labirua A, Lundberg IE. Interstitial lung disease and idiopathic inflammatory myopathies: progress and pitfalls. Curr Opin Rheumatol 2010;22:633-8.

3. Kato S, Sekine A, Kusakawa Y, Ogura T, Futaki M, Iwasawa T, et al. Prognostic value of cardiovascular magnetic resonance derived right ventricular function in patients with interstitial lung disease. J Cardiovasc Magn Reson 2015;17:10.

4. Zhang X, Wei X, Liang Y, Liu M, Li C, Hong T. Differential changes of left ventricular myocardial deformation in diabetic patients with controlled and uncontrolled blood glucose: a three-dimensional speckle-tracking echocardiography-based study. J Am Soc Echocardiogr 2013;26:499-506.

5. Vitarelli A, Martino F, Capotosto L, Martino E, Colantoni C, Ashurov R, et al. Early myocardial deformation changes in hypercholesterolemic and obese children and adolescents: a 2D and 3D speckle tracking echocardiography study. Medicine 2014;93:e71.

6. Galderisi M, Esposito R, Schiano-Lomoriello V, Santoro A, Ippolito $\mathrm{R}$, Schiattarella $\mathrm{P}$, et al. Correlates of global area strain in native hypertensive patients: a three-dimensional speckle-tracking echocardiography study. Eur Heart J Cardiovasc Imaging 2012;13:730-8.

7. Schwartz T, Diederichsen LP, Lundberg IE, Sjaastad I, Sanner H Cardiac involvement in adult and juvenile idiopathic inflammatory myopathies. RMD Open 2016;2:e00291.

J Rheumatol 2018;45:2; doi:10.3899/jrheum.170686 